\title{
Investigation of the vibration state of the turbine rotor taking into account the influence of geometric accuracy of parts
}

\author{
Michail Bolotov ${ }^{1}$, Vadim Pechenin ${ }^{1, *}$, and Ekaterina Kolchina ${ }^{1}$ \\ ${ }^{1}$ Samara University, Institute of Engines and Power Plants, 443086 Samara, Russian Federation
}

\begin{abstract}
The article describes the method of estimating the state of vibration of the rotor taking into account the geometrical deviations parts. The results obtained theoretical studies of the relationship of vibration characteristics and geometrical deviations of the rotor of low pressure turbine. To conduct research, a parametric rotor model has been created, as well as a finite element model in the ANSYS. To determine the center of mass of a part in the process of balancing, an algorithm developed in the program MATLAB.
\end{abstract}

\section{Introduction}

The quality of aviation products depends to a large extent on the technological processes of their manufacture. The achieved quality indicators of aviation products are measured at the stages of manufacturing, assembly and testing. The greatest complexity, high costs of time and money arise when achieving such quality indicators of products, which are measured in the late stages of product manufacturing: assembly and testing. Eliminating the identified inconsistencies in the late stages of product manufacturing often leads to the need to reexecute the previous steps. For example, detection of increased vibration of the product during the testing phase leads to the need for disassembly, control of parts and assembly units, modification or manufacture of parts, reassembly and subsequent testing. One of the reserves to improve the quality of aviation products in production is the use of digital technologies [1]. In the process of ensuring the quality of products during their production, digital counterparts of products can be used, which with sufficient accuracy reflect their key parameters. Forecasting the quality of products based on measured geometric data on parts and assembly units will allow to identify deviations before the tests and to formulate measures for their elimination.

One of the significant indicators of the quality of aviation products is its vibrational state, which depends on the achieved geometric accuracy of parts and assembly units, their elastic-inertial characteristics and the accuracy of dynamic balancing. The vibrational state of the rotor is affected by a variety of geometric deviations: the nonconcentricity of cylindrical surfaces relative to the geometric axis of rotation; beating of seats; the difference in the beads, the beating of the outer and inner surfaces relative to the axis of

${ }^{*}$ Corresponding author: v.a.pechenin@ssau.ru 
rotation, and the difference along the axis of the shaft; inaccurate assembly of aircraft engine assemblies [2].

To date, many works are known in which methods and models for assessing the vibrational state of rotors for the design phase [3-5]. The actual task is the development of methods and models for predicting the vibrational state of the turbine rotor, taking into account the influence of geometric parameters and the complexity of the problem and intended for use in technological purposes at the stages of manufacturing and assembling products. The development of such techniques and models will allow the creation of digital duplicates of products.

In the presented work results of researches on influence of geometrical deviations of details and assembly units on occurrence of the raised vibrations of a rotor.

\section{Technique of vibration state research}

The presented method is intended for evaluation of the rotor unbalances values depending on its geometrical deviations made during the manufacture and assembly. The description of the technique will be given in the example of a low-pressure turbine, for which 3-D models will be created, taking into account geometric deviations. The methodology uses the software package ANSYS. Flowchart developed technique shown in figure 1.

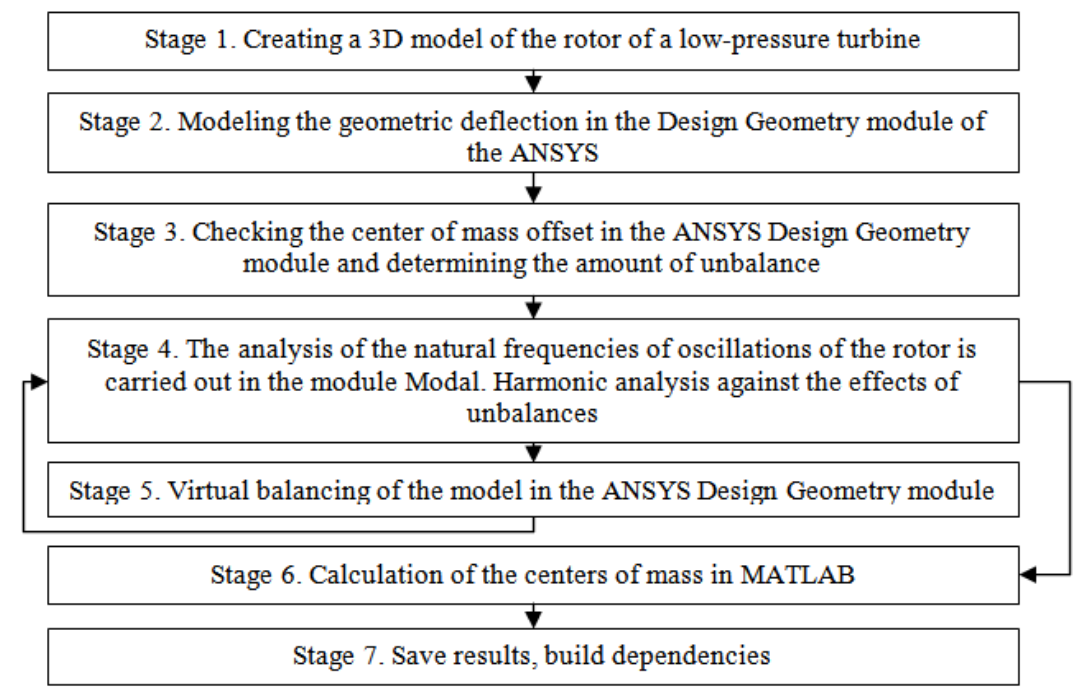

Fig. 1. Method for studying the vibrational state of the turbine rotor

The method includes, in aggregate, two parts. The first part prepares the geometry of the products for further calculations. In the second part, calculations are performed using the geometry of the product. We will describe the steps in the method.

The first stage is performed creating 3-D models assembly unit - the low pressure turbine. At the second stage, the CAD model is imported into the ANSYS software package, which simulates the considered geometric deviations. At production, the geometric deviations of the assembly can be checked with sufficient accuracy on the coordinate measuring machines [6]. In the third stage, the ANSYS Design Geometry module calculates the center of mass and determines the magnitude of the model unbalance before performing calculations.

At the fourth stage, the analysis of natural frequencies and subsequent harmonic analysis from the effect of unbalances in the Modal module of ANSYS. Boundary 
conditions are set that mimic the fastening of the rotor in a parametric form. The finite element mesh is superimposed. The general requirements for the grid are ordering (if possible, use only hexagonal elements), the minimum number of elements in the thickness of 3-4. To fulfill this condition, at the first stage a simplified turbine model is constructed that includes a shaft, spacer and a disk (figure 2).

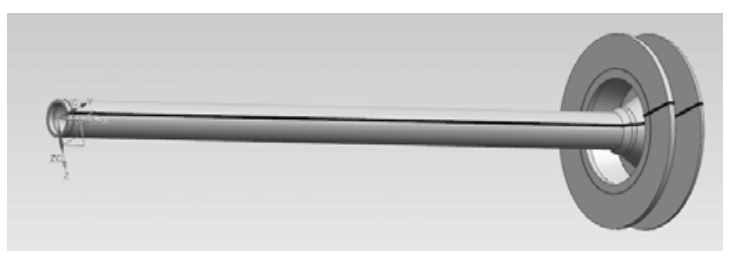

Fig. 2. A computational 3D model that takes into account geometric deviations.

For the given model, the setting of bearing installation sites, stiffness and damping coefficients, characterizing supports. Based on the results of the calculations, a Campbell diagram is constructed to determine the critical rotation frequencies. For the analysis of critical frequencies, a harmonic analysis is performed from the effect of unbalances. The graphs of the amplitude-frequency oscillations of the rotor and the deformed geometry in the *.stl. In the fifth stage, the initial unbalance of the rotor caused by geometric deviations is balanced with the use of a developed scroll that performs the selection of a virtual balancing weigher. The fourth step is again performed using a virtually balanced rotor model. At the sixth stage, the stl files are imported into the MATLAB system to calculate the center of mass and the magnitude of the unbalance. At the seventh stage, the results of the calculations are saved, the dependencies are constructed.

Let us consider in detail the developed algorithm for calculating the center of mass of the stl model.

\subsection{Algorithm for calculating the center of mass}

The *.stl file contains the following data: 1) $\mathbf{V}_{\mathrm{g} \times 3}$ (matrix of the coordinates of the vertices of the grid of the stl-model); 2) $\mathbf{F}_{\mathrm{m} \times 3}$ (matrix of combinations of vertices of three that form facets of surfaces); 3$) \mathbf{N}_{\mathrm{m} \times 3}$ (matrix of coordinates of normal facets).

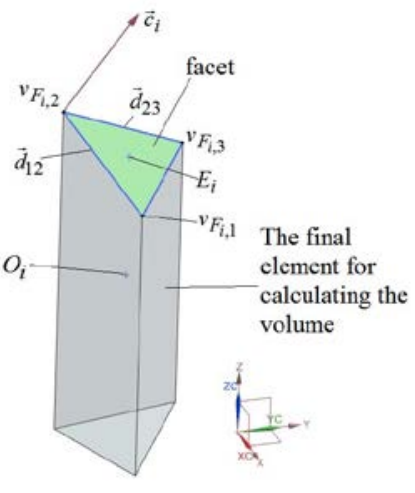

a)

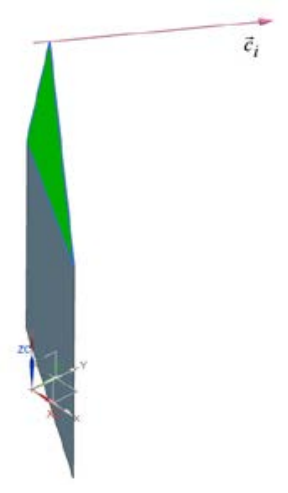

b)

Fig. 3. Construction of finite elements for calculating the volume and center of mass of the body a) the general case of $b$ ) the facet is perpendicular to XOY. 
The center of mass can be calculated using the volume of the body, equal in turn to the sum of the volumes of each individual element of the model. The body in the * ${ }^{*}$.stl format is a bound facet that describes the faces of the body. Facets are flat figures, each one does not form a three-dimensional body (every three vertices of the surface form facets).

Proceeding from this, it is necessary to supplement the existing facets to a three-dimensional shape. One option is to build facet projections onto the XOY coordinate plane and construct triangular truncated prisms (fig. 2, a). If the facet lies in a plane perpendicular to the XOY plane, then the volume of the resulting prism is zero (fig. $2, \mathrm{~b}$ ).

Having found the volumes of the obtained truncated prisms and the centers of mass of each prism, one can calculate the center of mass of the body. Assuming that the density of the material of the part is the same, the coordinates of the center of mass can be calculated on the basis of expressions:

$$
\left\{\begin{array}{l}
x_{c}=\left(\sum_{i=1}^{N} x_{O_{i}} \cdot V l_{i}\right) / V o l_{\text {sum }} \\
y_{c}=\left(\sum_{i=1}^{N} y_{O_{i}} \cdot V o l_{i}\right) / V o l_{\text {sum }} \\
z_{c}=\left(\sum_{i=1}^{N} z_{O_{i}} \cdot V o l_{i}\right) / V o l_{\text {sum }}
\end{array}\right.
$$

where $\left(x_{O_{i}}, y_{O_{i}}, z_{O_{i}}\right)$ - coordinates of the center of mass $O$ for $\mathrm{i}$-th finite element (truncated prism), forming the body (fig. 2,a);

$\mathrm{Vol}_{i}$ - volume of the i-th finite element;

$\mathrm{Vol}_{\text {sum }}$ - total body volume.

The volume of the $\mathrm{i}$-th finite element is calculated using the divergence theorem. Let's give a sequence of calculation of the center of mass of the body, given in the stl format.

1 . Calculation of the coordinates of the vectors forming the plane of the i-th trianglefacet $\vec{d}_{12}$ and $\vec{d}_{23}$ through the coordinates of the facet vertices $v_{F_{i, 1}}, v_{F_{i, 2}}$ and $v_{F_{i, 3}}$.

2. Computing the coordinates and modulus of a vector $\vec{c}_{i}$, normal to the plane of the i-th facet by means of a vector product $\vec{d}_{23}$ и $\vec{d}_{12}$.

3. The module of the vector product is equal to the area of the parallelogram constructed on the vectors $\vec{d}_{23}$ and $\vec{d}_{12}$, and The area of the triangle of the surface is half the area of this parallelogram. The area of the $i$-th triangle $s_{i}$ is calculated in this way using expression:

$$
s_{i}=0,5 \cdot\left|c_{i}\right|
$$

4. Calculating the $z$ coordinate of the midpoint of the facet $E_{i}$ :

$$
z_{\text {mean }_{i}}=\left(v_{F_{i, 1}, 1}+v_{F_{i, 1}, 2}+v_{F_{i, 3}, 3}\right) / 3 \text {. }
$$

5. The calculation of the volume of each finite element (formed from the facet) of the body:

$$
\operatorname{Vol}_{i}=s_{i} \cdot z_{\text {mean }_{i}} \cdot c_{n_{i}}(3),
$$


where $c_{n_{i}}(3)$ - component $z$ of the vector reduced to the norm.

6. Calculation of total volume $\mathrm{Vol}_{\text {sum }}$ as the sum of volumes $\mathrm{Vol}_{i}$.

7. Coordinates of the center of mass $O$ of the $i$-th truncated prism are calculated as:

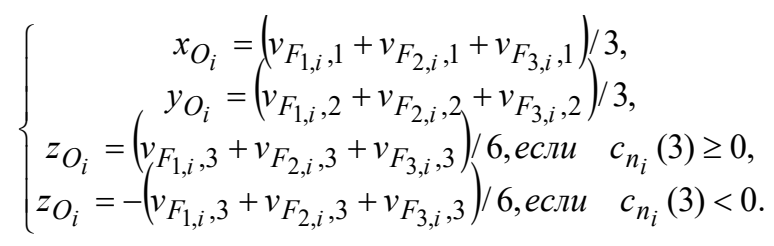

8. The calculation of the center of mass of the body is carried out by (1).

The developed algorithm was implemented in the software package MATLAB.

\subsection{Calculation of unbalance}

The unbalance of the rotor is calculated by the formula:

$$
U=m \cdot|\vec{r}|
$$

where $m$ - unbalanced weight, $\mathrm{kg}$;

$|\vec{r}|-$ module of center of gravity mass and rotation axis, $\mathrm{mm}$;

\section{Results}

Figure 4 shows the values of the unbalances of the rotor calculated according to the developed procedure (fig. 1). The geometric deviation in the experiments is the displacement of the internal shaft hole.

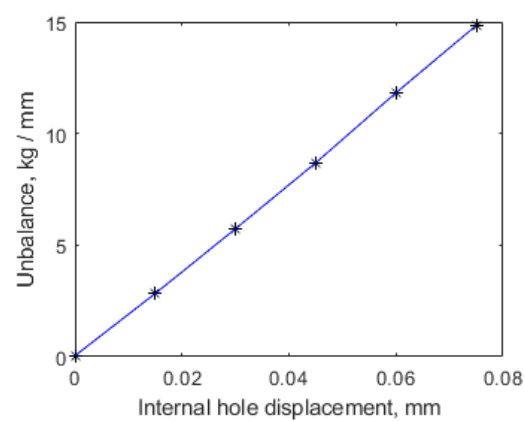

a

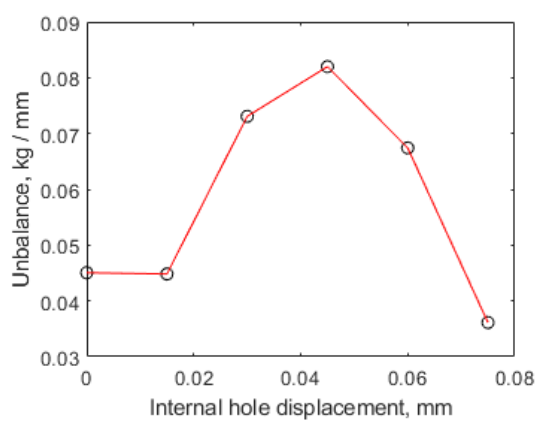

b

Fig. 4. Dependence unbalance of internal bore offset value obtained from the simulation before balancing (a) and after balancing(b).

From the simulation results it follows that the magnitude of the unbalance increases almost linearly with increasing displacement. After balancing, the unbalance decreases and becomes close to the unbalance on the nominal model. 


\section{Conclusion}

The presented work is devoted to the development of a method for predicting the vibrational state of a turbine rotor, taking into account the influence of geometric parameters. The resulted technique can be used in structure of digital doubles of products for use in the technological purposes at stages of manufacturing and assembly of products. On the basis of the above methodology, the relationship between the predicted values of the rotor unbalances and its geometric deviations. The results of the theoretical approbation of the methodology make it possible to conclude that it can be further developed with the purpose of industrial introduction to ensure the vibrational state of products.

This work was supported by the Ministry of Education and Science of the Russian Federation in the framework of the implementation of the Program State Assignment for 2018. The project code is 9.11978.2018/11.12.

\section{References}

1 A.V. Balaykin, K.A.Bezsonov, M.V. Nekhoroshev, , A.P. Shulepov, Developing Parametric Models for the Assembly of Machine Fixtures for Virtual Multiaxial CNC Machining Centers, IOP Conference Series: Materials Science and Engineering, v. 302(1), 012009 (2018)

2 M. E. Levit, V. P. Rojzman. Vibration and balancing of rotors of aircraft engines (Mashinostroenie, Moskow, 1970).

3 D. Kolmakova, G.Popov, A. Shklovets, A. Ermakov, Techniques and methods to improve the dynamic strength of gas turbine engines compressor rotor wheels, ASME 2014 Gas Turbine India Conference, GTINDIA 2014 (2014)

4 N.D. Kuznetsov, L.I. Fridman, A.I. Ermakov, V.N. Ukhov, Construction of dynamic designs for engine components based on equations of the theory of elasticity, Strength of Materials, v. 21(3), p. 267-274 (1989)

5 A.I. Ermakov, V.P. Ivanov, V.A. Frolov, Computation of the natural vibration frequencies of impellers in gas turbine engines on the basis of the method of dynamic wave stiffnesses and flexibilities, Strength of Materials, v. 20(6), p. 804-810 (1988)

6 A.F. Rezchikov, A.V. Kochetkov, O.V. Zakharov, Mathematical models for estimating the degree of influence of major factors on performance and accuracy of coordinate measuring machines, MATEC Web of Conferences, v. 129, 01054 (2017) 